\title{
UAS TOPOGRAPHIC MAPPING WITH VELODYNE LiDAR SENSOR
}

\author{
G. Jozkow ${ }^{\mathrm{a}, \mathrm{b}}, *, \mathrm{C}$. Toth ${ }^{\mathrm{a}}$, D. Grejner-Brzezinska ${ }^{\mathrm{a}}$ \\ ${ }^{a}$ Department of Civil, Environmental and Geodetic Engineering, The Ohio State University, 470 Hitchcock Hall, 2070 Neil Ave., \\ Columbus, OH 43210, USA - (jozkow.1, toth.2, grejner-brzezinska.1)@osu.edu \\ ${ }^{\mathrm{b}}$ Institute of Geodesy and Geoinformatics, Wroclaw University of Environmental and Life Sciences, Grunwaldzka 53, 50-357 \\ Wroclaw, Poland - grzegorz.jozkow@igig.up.wroc.pl
}

Commission I, ICWG I/Vb

KEY WORDS: UAS, UAV, LiDAR, mapping, performance analysis

\begin{abstract}
:
Unmanned Aerial System (UAS) technology is nowadays willingly used in small area topographic mapping due to low costs and good quality of derived products. Since cameras typically used with UAS have some limitations, e.g. cannot penetrate the vegetation, LiDAR sensors are increasingly getting attention in UAS mapping. Sensor developments reached the point when their costs and size suit the UAS platform, though, LiDAR UAS is still an emerging technology. One issue related to using LiDAR sensors on UAS is the limited performance of the navigation sensors used on UAS platforms. Therefore, various hardware and software solutions are investigated to increase the quality of UAS LiDAR point clouds. This work analyses several aspects of the UAS LiDAR point cloud generation performance based on UAS flights conducted with the Velodyne laser scanner and cameras. The attention was primarily paid to the trajectory reconstruction performance that is essential for accurate point cloud georeferencing. Since the navigation sensors, especially Inertial Measurement Units (IMUs), may not be of sufficient performance, the estimated camera poses could allow to increase the robustness of the estimated trajectory, and subsequently, the accuracy of the point cloud. The accuracy of the final UAS LiDAR point cloud was evaluated on the basis of the generated DSM, including comparison with point clouds obtained from dense image matching. The results showed the need for more investigation on MEMS IMU sensors used for UAS trajectory reconstruction. The accuracy of the UAS LiDAR point cloud, though lower than for point cloud obtained from images, may be still sufficient for certain mapping applications where the optical imagery is not useful.
\end{abstract}

\section{INTRODUCTION}

Unmanned Aerial Systems (UASs) are getting recently more interest in the mapping community. They are offering similar products as traditional (manned) airborne systems but at lower costs. The current practice of UAS mapping is based on the imagery data that is indirectly georeferenced using Ground Control Points (GCPs). However, some applications need active sensors (e.g. LiDAR) that require direct georeferencing approach using navigation sensor data.

The navigation sensors, such as Global Navigation Satellite System (GNSS) receivers and Inertial Measurement Units (IMUs) that are used for controlling and navigating typical Unmanned Aerial Vehicles (UAVs) are of lower performance and are not able to provide enough accurate georeferencing data. High grade IMUs might be used only for large UAS (Yang and Chen, 2015) what significantly increases system weight and costs. In the case of small UAS (sUAS), typically low grade micro-electro-mechanical systems (MEMS)-based IMUs are suitable and are frequently used for navigating and stabilizing the UAS platform. MEMS technology allowed for the significant reduction of the cost, weight, size, and power requirements of IMU sensors, but at the price of the performance, since such IMUs are characterized by large bias and noise (El-Sheimy, 2009). With strong developments in MEMS sensor technology, the newest IMUs are approaching the tactical grade performance, which is the dominant grade in typical aerial mapping systems. Some works have already proved that MEMS IMU might support UAS mapping (Hirose et al., 2015; Tulldahl and Larsson, 2014).

In the case of GNSS receivers and antennas, UAS platforms are usually equipped with single-frequency Global Navigation System (GPS) receivers which are of sufficient performance for navigating the platform, but not for mapping purposes. However, a high (geodetic) grade miniaturized multi GNSS receivers are already available on the market at a reasonable price, and can be easily carried on even by the sUAS. These receivers very often support Real Time Kinematic (RTK) positioning that can be potentially used in real time applications. However, the drawback of the RTK is that it requires stable data linkage (e.g. through radio modem) and the computed position cannot be improved in post-processing since the raw observations are unavailable.

The variety of the LiDAR sensors that are suitable for UAS is also growing. The first scanners used for that purpose, e.g. SICK LMS-291 were of relatively low performance, and their weight required the use of very heavy platforms (Nagai et al., 2009). The improvement in the LiDAR technology resulted in the reduction of weight and thus light-weight sensors, such as the ibeo LUX and SICK LD-MRS-400001 (Line et al. 2011; Wallace et al., 2012), and ultra-light weight $(0.2 \mathrm{~kg})$ Hokuyo UTM-30LX scanner (Kuhnert and Kuhnert, 2013; Roca et al., 2014; Hirose et al., 2015) were used on UAS platforms. However, these sensors were still of low performance. The first high performance laser scanner dedicated for UASs is the Riegl UAVX-1UAV (Amon et al., 2015; Mandlburger et al., 2015). In

* Corresponding author 
contrast to other UAS scanners, the Riegl UAVX-1UAV is characterized by very long range measurements (up to $920 \mathrm{~m}$ ). The good performance, however, comes with larger weight (3.6 $\mathrm{kg}$ ) that limits the use of this sensor only to larger platforms. Due to the use of multiple laser diodes (channels) inside a single LiDAR sensor, Velodyne scanners are gaining attention in UAS mapping, in particular the Velodyne HDL-32E (Tulldahl and Larsson, 2014; Zhe et al., 2015) and the new Velodyne VLP-16. Although the range of these sensors is not very high, the data acquisition rate, low weight and price make these scanners very attractive to use in UAS topographic mapping.

This work investigates the performance of a UAS equipped with Velodyne laser scanner, where the platform direct georeferencing is based on the MEMS IMU and dual-frequency miniaturized GPS receiver data. The optical imagery obtained by the same platform is used for comparison purposes and to support alternative approaches of the platform georeferencing.

\section{MATERIALS}

\subsection{Test UAS}

The platform used in this study is the multipurpose Bergen octocopter equipped with variety of navigation sensors (Figure 1), including:

1. NovAtel OEM615 dual-frequency GPS receiver and Antcom antenna

2. Garmin GPS-18LV single-frequency GPS receiver and antenna for time tagging the Velodye LiDAR data

3. Solmeta Geotagger N3 single-frequency GPS receiver and antenna for position tagging of Nikon camera images

4. Wookong-M autopilot systems consisting of singlefrequency GPS receiver and antenna, MEMS IMU, and magnetometer; used only for navigating and controlling octocopter flight

5. MicroStrain 3DM-GX3-35 consisting of singlefrequency GPS receiver and antenna, MEMS IMU, and magnetometer; GPS sensor was used only for time tagging IMU data; magnetometer data was not used in this study

6. Epson M-G362PDC1 MEMS IMU

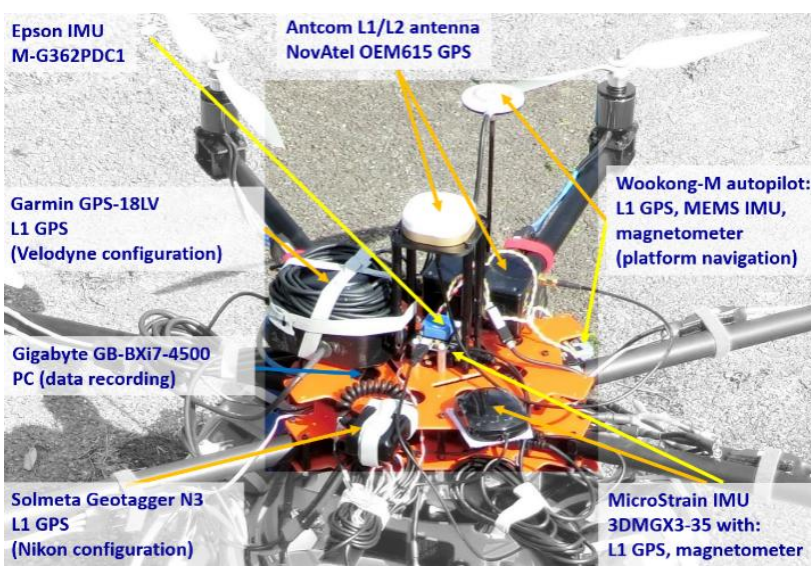

Figure 1. Navigation sensors mounted on the Bergen octocopter

The platform was also equipped with Gigabyte GB-BXi7 4500 small computer for recording data obtained from the Velodyne laser scanner, NovAtel GPS receiver, and MicroStrain and Epson IMUs. Besides collecting GPS raw observations, the
NovAtel receiver was also used for time tagging Epson IMU data, and recording GPS time stamps for Nikon camera images. The two MEMS IMUs mentioned above are approaching the tactical grade performance level (Table 1) that should result in the data of sufficient accuracy for the integration with the GPS data acquired by the NovAtel receiver, and subsequently, for direct georeferencing. The two IMUs were used for comparison purposes. Note that according to the specification (Table 1) Epson IMU has better gyros (lower value of gyro bias stability) and slightly worse accelerometers than the MicroStrain IMU.

\begin{tabular}{|c|c|c|}
\hline IMU & $\begin{array}{c}\text { Epson } \\
\text { M-G362PDC1 }\end{array}$ & $\begin{array}{c}\text { MicroStrain } \\
\text { 3DM-GX3-35 }\end{array}$ \\
\hline $\begin{array}{l}\text { Gyros } \\
\text { Measurement range }[\% / \mathrm{s}] \\
\text { In-run bias stability }[\% / \mathrm{h}] \\
\text { Initial bias error }[\% / \mathrm{s}] \\
\text { Noise density }\left[\% / \mathrm{s} / \mathrm{Hz}^{1 / 2}\right]\end{array}$ & $\begin{array}{l} \pm 150 \\
3 \\
\pm 0.5 \\
0.002\end{array}$ & $\begin{array}{l} \pm 300 \\
18 \\
\pm 0.25 \\
0.03\end{array}$ \\
\hline $\begin{array}{l}\text { Accelerometers } \\
\text { Measurement range }[g] \\
\text { In-run bias stability }[\mathrm{mg}] \\
\text { Initial bias error }[\mathrm{m} g] \\
\text { Noise density }\left[\mathrm{m} g / \mathrm{Hz}^{1 / 2}\right]\end{array}$ & $\begin{array}{l} \pm 3 \\
<0.1 \\
\pm 8 \\
0.1\end{array}$ & $\begin{array}{c} \pm 5 \\
0.04 \\
\pm 2 \\
0.08\end{array}$ \\
\hline $\begin{array}{l}\text { Other } \\
\text { Power consumption [mA] } \\
\text { Net weight [gram] }\end{array}$ & $\begin{array}{c}30 \\
7\end{array}$ & $\begin{array}{c}200 \\
23\end{array}$ \\
\hline
\end{tabular}

Table 1. Parameters of used IMU sensors

Since the used Bergen octocopter is a multipurpose platform, it can support variety of mapping sensors. In this study, four sensors are investigated, including:

1. Velodyne HDL-32E profile laser scanner

2. Velodyne VLP-16 profile laser scanner

3. Nikon D800 camera with Nikon a Nikkor AF-S 50 $\mathrm{mm} \mathrm{f} / 1.4 \mathrm{G}$ lens

4. GoPro Hero 3+ Black Edition camera with non-stock $5.4 \mathrm{~mm}$ lens

\begin{tabular}{|c|c|c|}
\hline Velodyne laser scanner & HDL-32E & VLP-16 \\
\hline \multicolumn{3}{|l|}{ Appearance } \\
\hline Channels & 32 & 16 \\
\hline Max. operation range $[\mathrm{m}]$ & 100 & 100 \\
\hline Accuracy $[\mathrm{cm}]$ & \pm 2 & \pm 3 \\
\hline Dual returns & yes & yes \\
\hline Horizontal field of view $\left[^{\circ}\right]$ & 360 & 360 \\
\hline Horizontal resolution $\left[{ }^{\circ}\right]$ & $\begin{array}{l}0.08 @ 5 \\
0.16 @ 10\end{array}$ & $\begin{array}{l}0.1 @ 5 \\
02 @ 10\end{array}$ \\
\hline @ rotation rate $[\mathrm{Hz}]$ & $0.35 @ 20$ & $0.4 @ 20$ \\
\hline Vertical field of view $\left[{ }^{\circ}\right]$ & -30.67 to +10.67 & -15 to +15 \\
\hline Vertical resolution $\left[{ }^{\circ}\right]$ & 1.3 & 2.0 \\
\hline Measurement rate [pts/s] & $\sim 700,000$ & $\sim 300,000$ \\
\hline Power consumption [W] & 12 & 8 \\
\hline Net weight $[\mathrm{kg}]$ & 1 & 0.8 \\
\hline
\end{tabular}

Table 2. Parameters of two Velodyne sensors

Tables 2 and 3 list the parameters of investigated LiDAR and imagery sensors, respectively. The performance of both LiDAR 
sensors is similar, however the HDL-32E can acquire more data resulting in higher density, but VLP-16 is less heavy and does not contain outer moving parts.

\begin{tabular}{|l|c|c|}
\hline Camera & Nikon & GoPro \\
\hline Appearance & & \\
& & \\
Sensor width @ height $[\mathrm{mm}]$ & 35.9 @ 24.0 & $6.2 @ 4.65^{\mathrm{a}}$ \\
Image width @ height $[\mathrm{pix}]$ & 7360 @ 4912 & 4000 @ 3000 \\
Pixel size $[\mu \mathrm{m}]$ & $\sim 4.88$ & 1.55 \\
Focal length $[\mathrm{mm}]$ & 50 & 5.4 \\
Horizontal field of view $\left[^{\circ}\right]$ & 39 & 60 \\
Weight $[\mathrm{kg}]$ & 1.3 & 0.076 \\
\hline
\end{tabular}

${ }^{\text {a) }}$ calculated from pixel and image size

Table 3. Parameters of the used cameras

Obviously, the payload of the used platform is limited and the octocopter cannot be equipped with all aforementioned sensors at the same time. However, besides the flight with one sensor at a time, the size and weight of the Velodyne VLP-16 and GoPro camera allow to mount them simultaneously and perform the flight. Note that the used UAS in the heaviest configuration did not exceed $13 \mathrm{~kg}$ of the total takeoff weight (platform, sensors, power supply, etc.). The span of the Bergen octocopter is $1 \mathrm{~m}$.

\subsection{Test Flights}

Test flights were executed in a waypoint based mode over relatively flat terrain containing moderate complexity of nonground objects. Although the plan (Figure 2) was identical for all flights, the actual flight trajectories may differ due to lower performance of the navigation sensors included in the autopilot system. Table 4 lists parameters that are common for all flights, and nominal parameters specific for each mapping sensor.

\begin{tabular}{|c|c|}
\hline $\begin{array}{l}\text { Common parameters } \\
\text { Flying altitude AGL }[\mathrm{m}] \\
\text { Flying speed }[\mathrm{m} / \mathrm{s}] \\
\text { Number of strips } \\
\text { Length of the strip }[\mathrm{m}] \\
\text { Distance between strips [m] } \\
\text { NovAtel data acquisition rate }[\mathrm{Hz}] \\
\text { Minimum IMU data acquisition rate }[\mathrm{Hz}]\end{array}$ & $\begin{array}{c}25 \\
4 \\
3 \\
73.72 \\
12.57 \\
5 \\
100\end{array}$ \\
\hline $\begin{array}{l}\text { Parameters for } \\
\text { Nikon / GoPro flights } \\
\text { Image acquisition rate }[\mathrm{Hz}] \\
\text { GSD at nadir [mm] } \\
\text { Footprint width [m] } \\
\text { Footprint height [m] } \\
\text { Image endlap [\%] } \\
\text { Image sidelap [\%] }\end{array}$ & $\begin{array}{c}1 / 2 \\
12 / 2.5 \\
17.95 / 28.70 \\
12.00 / 21.53 \\
67 / 93 \\
30 / 56\end{array}$ \\
\hline $\begin{array}{l}\text { Parameters for Velodyne } \\
\text { HDL-32E / VLP-16 flights } \\
\text { Rotation rate }[\mathrm{Hz}] \\
\left.\text { Across flight FOV }{ }^{\circ}\right] \\
\text { Swath width [m] } \\
\text { Strip overlap [\%] } \\
\text { Average point spacing across flight }{ }^{\mathrm{a})}[\mathrm{m}] \\
\text { Average point spacing along flight }{ }^{\mathrm{a})}[\mathrm{m}] \\
\text { Along flight overlap [\%] } \\
\text { Nominal average point density }\left[\mathrm{pts} / \mathrm{m}^{2}\right]\end{array}$ & $\begin{array}{c}10 \\
60 \\
28.87 \\
56 \\
0.08 / 0.10 \\
0.4 \\
98 / 97 \\
1,039 / 416 \\
\end{array}$ \\
\hline
\end{tabular}

${ }^{\text {a) }}$ for single $0^{\circ}$ channel

Table 4. Parameters of the flight and data acquisition

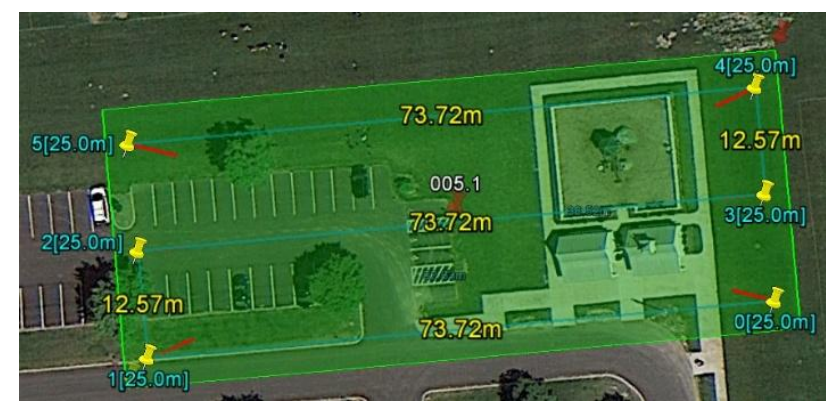

Figure 2. Test flights plan

The cameras were mounted on the platform to take vertical images; Nikon camera was fixed to the octocopter body while GoPro camera was mounted on a gyro-stabilized gimbal. For that reason, the actual tilt angle of the Nikon camera was dependent on the platform attitude. Similarly, Velodyne scanners are fixed to the platform with rotation axis aligned with the octocopter nominal movement direction (Figure 3). Since each of the Velodyne scanners has more than a single laser diode, during each rotation a pattern of 32 or 16 lines (depending on the scanner) is created. The consecutive patterns overlap, and for the particular parameters (Table 4), this overlap is equal $98 \%$ or $97 \%$, depending on the scanner. This causes that actual point spacing along flight direction is much higher than if obtained by single channel sensors. Consequently, this overlap causes also higher nominal point cloud density. The difference in nominal point cloud densities for HDL-32 and VLP-16 is mainly due to the different number of sensor channels and slightly different horizontal resolution for the same scanning rate (Table 2).



Figure 3. Scanning principle of a horizontally mounted

Velodyne HDL-32E scanner on a UAS platform

To support direct georeferencing of the UAS platform, a GPS base station was established to collect dual-frequency GPS observations in the period of UAS flights. For indirect image georeferencing, a set of GCPs was measured with the average 3D accuracy of $2 \mathrm{~cm}$. Similarly, for accuracy assessment, a set of check points was surveyed in the field. A total number of measured ground points was 19 , including 10 signalized targets and 9 natural, easy to identify points. 


\section{METHODS}

\subsection{Dense Point Cloud Generation from Images}

The image based point cloud was generated by the commercial software that implements the standard workflow for UAS images processing. In particular, first the bundle block adjustment (BA) is applied (based on the GCPs) to find the position and orientation of each image, and then the dense image matching creates the dense point cloud.

\subsection{Georeferencing of LiDAR Point Cloud}

The generation of the georeferenced point cloud is the transformation from the scanner local coordinates (scanner frame $s$ ) to the global coordinates (frame $g$ ). The model of generating local coordinates from the Velodyne raw measurements for the channel $k$ is as follow (Glennie and Litchi, 2010):

$$
\left[\begin{array}{l}
x_{k} \\
y_{k} \\
z_{k}
\end{array}\right]^{S}=\left[\begin{array}{c}
\rho_{k} \cdot \cos \delta_{k} \cdot \sin \left(\varepsilon-\beta_{k}\right)-H_{k} \cdot \sin \left(\varepsilon-\beta_{k}\right) \\
\rho_{k} \cdot \cos \delta_{k} \cdot \cos \left(\varepsilon-\beta_{k}\right)+H_{k} \cdot \cos \left(\varepsilon-\beta_{k}\right) \\
\rho_{k} \cdot \sin \delta_{k}+V_{k}
\end{array}\right]
$$

where $\rho_{k}=r_{k} \cdot R_{k}+D_{k} \quad$ is the calibrated range measurement

$r_{k}=$ raw range measurement

$R_{k}=$ range scale factor

$D_{k}=$ range offset

$\delta_{k}=$ vertical angle correction

$\varepsilon=$ horizontal angle measurement encoder

$\beta_{k}=$ horizontal angle rotation correction

$H_{k}=$ horizontal offset from scanner origin

$V_{k}=$ vertical offset from scanner origin

The local scanner coordinates $s$ of the point acquired at a time $t$ are then georeferenced according to the model:

$$
\left[\begin{array}{l}
x(t) \\
y(t) \\
z(t)
\end{array}\right]^{g}=\left[\begin{array}{l}
X(t) \\
Y(t) \\
Z(t)
\end{array}\right]^{g}+R_{b}^{g}(t) \cdot\left(R_{S}^{b} \cdot\left[\begin{array}{l}
x(t) \\
y(t) \\
z(t)
\end{array}\right]^{s}+\left[\begin{array}{l}
l_{x} \\
l_{y} \\
l_{z}
\end{array}\right]^{b}\right)
$$

where

$$
\begin{aligned}
& {\left[\begin{array}{l}
X(t) \\
Y(t) \\
Z(t)
\end{array}\right]^{g}=\text { coordinates of the body frame origin given }} \\
& \text { in the mapping frame } g \\
& R_{b}^{g}(t)=f(r(t), p(t), h(t)) \text { is the rotation matrix } \\
& \text { from the body frame } b \text { to the mapping frame } g \text { that is } \\
& \text { dependent on the Euler angles: roll } r \text {, pitch } p \text {, and } \\
& \text { heading } h \\
& R_{s}^{b}=\text { rotation matrix from the scanner frame } s \text { to the } \\
& \text { body frame } b \text { that is dependent on the a priori rotation } \\
& \text { matrix based on the body frame definition and scanner } \\
& \text { assembly, and rotation caused by the boresight } \\
& \text { misalignment } \\
& {\left[\begin{array}{l}
l_{x} \\
l_{y} \\
l_{z}
\end{array}\right]^{\text {a lever-arm offset between scanner and body }}}
\end{aligned}
$$
frame origins defined in the body frame $b$

The Velodyne scanners are factory calibrated, i.e., all six calibration parameters (see Eq. (1)) for each scanner channel are known. In general, this simplifies georeferencing to the Eq. (2). The intra-sensor calibration parameters, such as lever-arm offsets between scanner and IMU origins or GPS antenna reference point and IMU origin are usually obtained by direct measurements and can be adjusted during processing. Similarly, boresight misalignment is usually assumed to be zero on the beginning and then can be adjusted during processing (Skaloud and Lichti, 2006).

The essential part of the point cloud georeferencing is the estimation of time dependent position and orientation of the body frame in the global/mapping frame. This task is known as platform direct georeferencing or trajectory reconstruction. The six parameters (3D position and attitude) are usually estimated by integrating GPS and IMU data where the body frame is chosen as the IMU frame. The state-of-the-art in GPS/IMU data integration is the Extended Kalman Filter (EKF); note that there are many filter designs and implementations. In particular, the integration can be executed in loosely or tightly coupled modes, and the filters can differ by the state vector length (Jekeli, 2001). In this work the integration was performed in looselycoupled mode with the EKF state vector length of 21, including 3 positions, 3 velocities, 3 orientation angles, 3 gyro biases, 3 accelerometer biases, 3 gyro scale factors, and 3 accelerometer scale factors.

\subsection{Accuracy Assessment}

The accuracy of the image based point cloud was evaluated using precisely measured ground reference points. Since the dense matching can provide points with RGB information for almost every pixel of the image, the point spacing for the test data was very high. Consequently, the identification of corresponding reference points was possible. Based on the coordinate residuals, both horizontal and vertical accuracies were calculated.

In the case of LiDAR point clouds, direct comparison with ground reference points is nearly impossible due to lower point cloud density, as ground targets may be difficult to find based on LiDAR intensity information and point coordinates. In addition, sparse data introduces additional error. For example, in our tests, the nominal average point spacing for Velodyne VLP-16 point cloud is about $5 \mathrm{~cm}$, and none of these points may be exact reflection from the reference point measured directly in the field. For that reason the accuracy of the point cloud must be estimated in a different manner.

Since the LiDAR point clouds are usually used for surface modeling, the vertical accuracy was estimated on the basis of the created Digital Surface Model (DSM). After removing noise points, the DSM of the GRID size equal $0.1 \mathrm{~m}$ was interpolated and compared against heights of ground reference points. Based on height residuals the vertical RMSE was calculated.

The surface modeling can also benefit in the estimation of the accuracy for horizontal and vertical components separately. Similarly to the evaluation of the image based point cloud described above, a comparison with ground reference points can be executed. However, points corresponding to reference are not selected directly in the point cloud, but must be estimated during a modeling process, e.g. as the intersection of modeled edges. Obviously, such approach requires appropriate objects that can be modeled from the point cloud in order to extract feature points. In addition, the accuracy estimated using this approach is affected by the accuracy of modeling, e.g. fitting surfaces to the point cloud, surface intersecting, etc. 
The point cloud based modeling of geometrical features can be used to assess an internal accuracy of both types of point clouds. From the georeferenced point cloud several subsets of points can be extracted that belong to flat surfaces (e.g. building roofs). Using least squares methods (LSQ), planar patches can be fitted to each set of points allowing for calculating residuals and other statistical parameters, including RMSE that can be treated as an accuracy estimate. To avoid estimating the planar patch parameters from outlier points, a robust LSQ may be applied. Obtained estimate is a measure of internal 3D accuracy (consistency) of the point cloud since selected planar patches usually have different orientations.

The relative comparison of two types of point clouds (LiDAR and imagery) can be also executed based on the planar patches. In this case LiDAR point residuals are computed with respect to planar patches estimated from the image point cloud. These values cannot be considered as absolute accuracy of the LiDAR point cloud, because the accuracy of the image point cloud, obtained even form high resolution and good geometry images may be too low to treat them as a reference. However, such approach can indirectly compare two types of point clouds.

LiDAR and image point clouds can be also compared directly. For well mapped objects, a set of corresponding point clouds can be extracted and the distance between LiDAR with respect to image based point cloud, treated as the reference, can be calculated. Obtained values may provide relative point cloud accuracy.

\section{RESULTS AND DISCUSSION}

Depending on the test flight, the GPS positions estimated from the NovAtel data showed 3D accuracy of $10 \mathrm{~cm}$ or better. The analysis of IMU data showed strong noise in accelerometer readings. Table 5 shows the mean accelerometer readings for two parts of the flight, including the system test before the takeoff; note that both IMUs were aligned to have the same orientation of $\mathrm{Z}$ axis (opposite polarity) and then the axis $\mathrm{X}$ of one IMU corresponded to the axis Y of the other IMU.

\begin{tabular}{|l|c|c|c|c|c|}
\hline \multirow{2}{*}{ Test case } & \multirow{2}{*}{ IMU } & \multicolumn{4}{|c|}{$\begin{array}{c}\text { Mean of accelerometer } \\
\text { readings }\left[\mathrm{m} / \mathrm{s}^{2}\right]\end{array}$} \\
\cline { 3 - 6 } & & Total & $\mathrm{X}$ & $\mathrm{Y}$ & $\mathrm{Z}$ \\
\hline Static & MicroStrain & 9.81 & 0.96 & 0.21 & -9.43 \\
(motors off) & Epson & 9.81 & 0.21 & 0.91 & 9.44 \\
\hline Inflight & MicroStrain & 8.88 & -0.02 & 0.38 & -8.28 \\
(motors on) & Epson & 9.72 & -0.57 & -0.22 & 8.49 \\
\hline
\end{tabular}

Table 5. Analysis of accelerometer readings for the flight with Velodyne HDL-32E

To integrate unreliable IMU data with relatively good GPS positions, the parameters of the EKF were tuned to account for the high acceleration noise. This approach caused that obtained positions were nearly identical to positions calculated from GPS data, and the calculated platform orientation was mainly dependent on gyro readings. In addition to the $6 \mathrm{DoF}$ trajectory reconstruction by GPS/IMU data integration, the $6 \mathrm{DoF}$ octocopter trajectory for one flight was obtained from imagery data as a result of BA. This allowed to see the impact of the noisy IMU data to the platform orientation.

Since properly executed indirect georeferencing is usually more accurate than direct georeferencing, the GCPs based BA solution based on the good geometry Nikon imagery was treated as a reference. Because the reference solution was estimated with $1 \mathrm{~Hz}$ rate, only the corresponding part of GPS/IMU solution was compared. The comparison of the GPS and reference BA trajectory points showed that the actual 3D accuracy of GPS positions with respect to reference BA solution was about $4 \mathrm{~cm}$. The analysis of the attitude (Figure 4) showed that the solution obtained from MicroStrain data is very similar to the reference, but the solution from Epson data is not reliable because it shows very large tilt angle (even $50^{\circ}$ ) that would cause the octocopter to crash if this tilt happens in the reality. For that reason, the direct georeferencing of the LiDAR point cloud was performed using the MicroStrain solution. The unreliable attitude obtained from Epson data may be explained by very strong influence of motor vibrations to Epson gyro readings.


Figure 4. Euler angles obtained from GSP/IMU data integration using modified EKF and Nikon images (reference) 


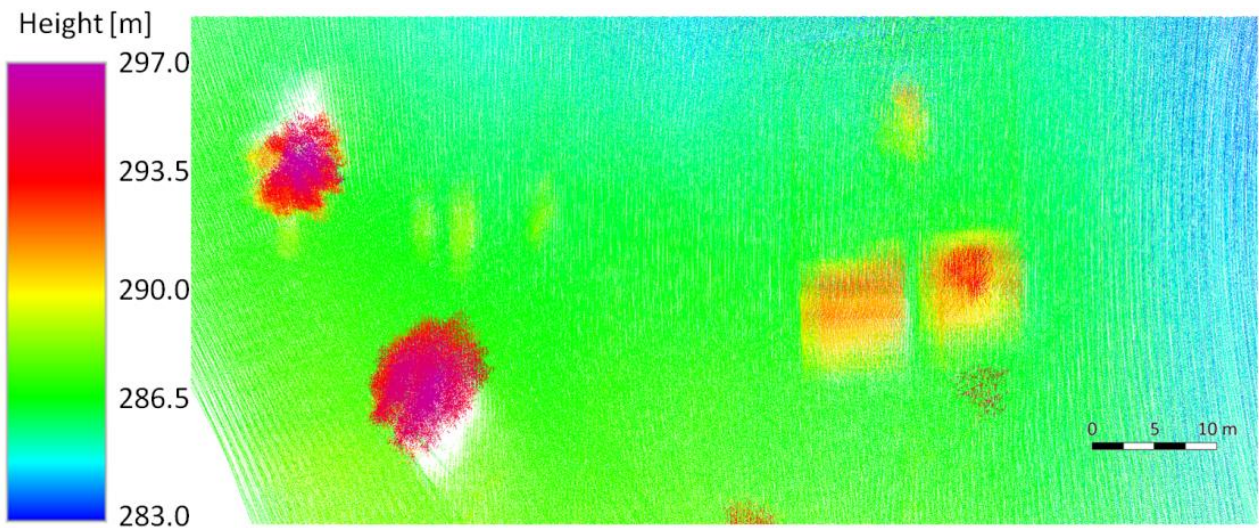

283.0

(a)

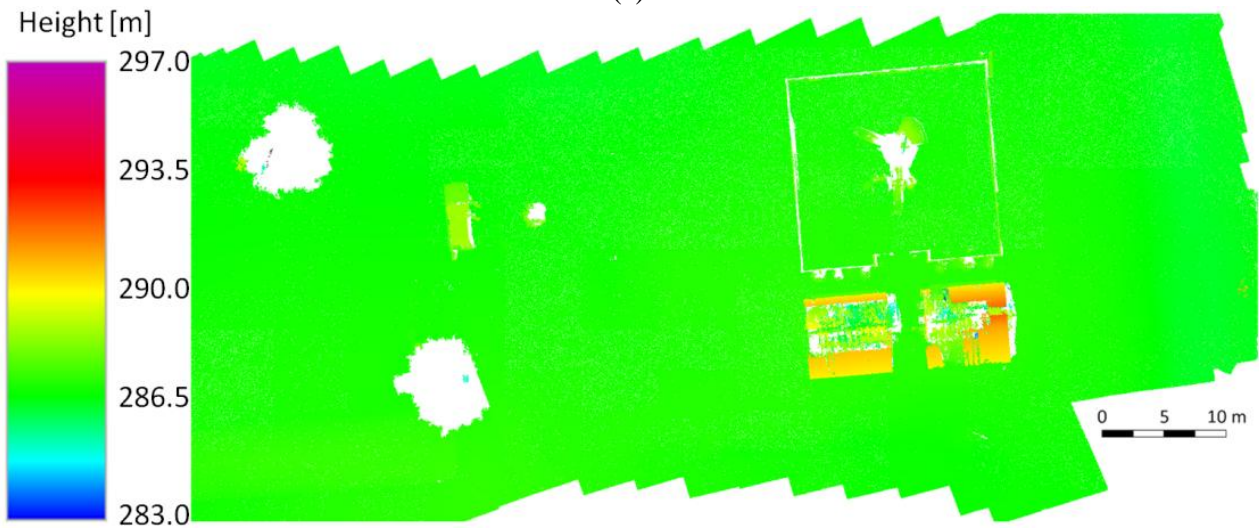

(b)

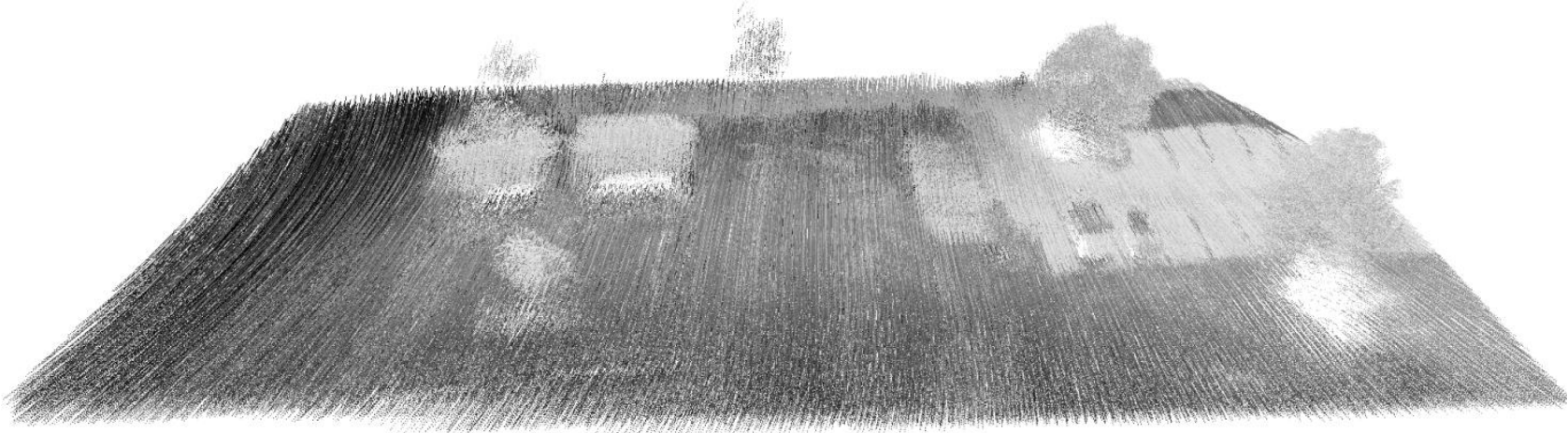

(c)

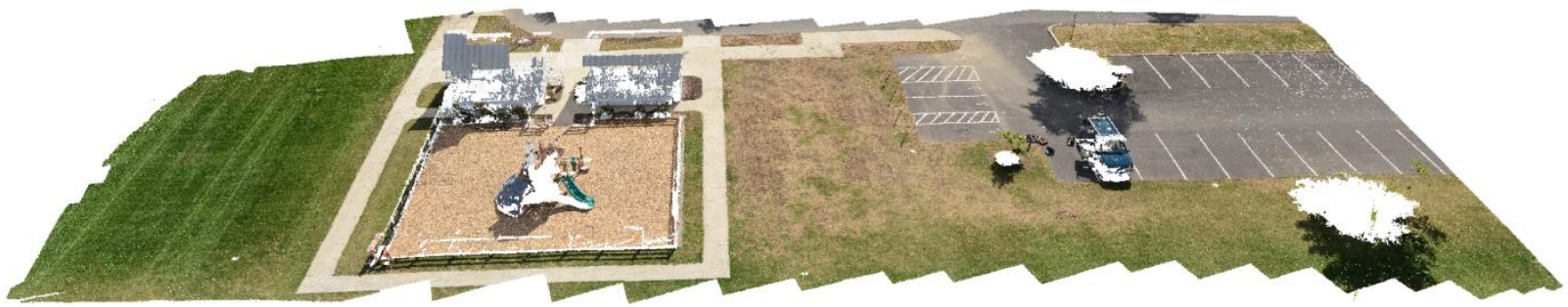

(d)

Figure 5. Obtained point clouds: Velodyne HDL-32E - heights (a), Nikon - heights (b), Velodyne HDL-32E - intensity (c), Nikon RGB (d)

Results of the georeferenced point clouds obtained from Velodyne HDL-32E scanner data and Nikon images are shown in Figure 5. Clearly, the LiDAR point cloud is more complete as it contains reflections from the objects that lack the texture necessary for dense image matching (roofs) and objects that were moving during data acquisition - wind caused the movement of tree branches. The Velodyne point cloud is also sparser than the point cloud obtained from Nikon images. The calculated point cloud density is 867 and $12,429 \mathrm{pts} / \mathrm{m}^{2}$ for the Velodyne HDL-32E and Nikon point clouds, respectively. However, the point cloud density for used LiDAR sensor is still much larger than obtained from traditional airborne LiDAR systems. The detailed analysis of the Velodyne point cloud geometry showed issues with the trajectory reconstruction, because many points were unaligned with flat surfaces (e.g. building roofs), and edges of the straight objects (e.g. curb 
separating parking lot from the lawn, and edges of buildings) were not straight. For that reason, only initial estimate of absolute vertical accuracy of created DSM was computed and was equal 0.49 and $0.03 \mathrm{~m}$ for Velodyne and Nikon point clouds, respectively. As reported earlier (Toth et al., 2015) the absolute 3D accuracy of Nikon point cloud is $7 \mathrm{~cm}$, this point cloud can be used as the reference in the relative comparison with the Velodyne point cloud. 3D distances between Velodyne and Nikon point clouds were calculated using the CloudCompare software (Girardeau-Montaut, 2016) resulting in the average distance of $0.90 \mathrm{~m}$; the standard deviation of these distances was $0.65 \mathrm{~m}$. Obtained values are rather large comparing to the accuracy of the Velodyne HDL-32E range measurements or the accuracy of positioning using geodetic grade GPS receiver. Further improvement of the UAS LiDAR point cloud requires more accurate estimation of the platform attitude.

\section{POSSIBILITIES OF LIDAR POINT CLOUD IMPROVEMENT}

\subsection{Trajectory Reconstruction with Imagery Data}

There are few aspects to improve the UAS Velodyne point cloud accuracy for the investigated hardware in this study. First is the reduction of the vibration impact on the IMU readings. This may be obtained by modifying the mount and adding dampers to isolate the IMU and scanner from the vibrating octocopter frame. Obviously, IMU and LiDAR sensor should not be isolated.

The use of multiple laser diodes in one sensor may be potentially beneficial in refinement of the platform attitude. Because all Velodyne pulses for the same horizontal angle are emitted nearly simultaneously, created profiles from different rotations may be matched adding constraints to the attitude estimation. However, this approach needs further algorithmic developments.

Finally, the $6 \mathrm{DoF}$ trajectory of the UAS can be estimated also from the imagery data without any data from navigation sensors. In this case, the position and orientation is computed for each taken image. This task can be solved by image block BA based on GCPs. Consequently, for the LiDAR point cloud georeferencing, the sensor body is the image frame instead of IMU frame. Such type of solution can be beneficial if the IMU data is not reliable, e.g. affected by the motor vibrations (Zhe et al., 2015). The requirement is that the LiDAR and imagery data needs to be acquired during the same flight. In the case of the testing platform, due to its limited payload capacity, only Velodyne VLP-16 and GoPro camera could be mounted at the same time on the octocopter.

Since the GoPro camera does not provide accurate time of image acquisition, the reconstructed points based on BA trajectory lack the time stamps. Obviously, the NovAtel GPS data is able to provide reliable time and position of the platform. The trajectories (3DoF) obtained from images and GPS data can be matched and interpolated to get the time dependent $6 \mathrm{DoF}$ trajectory and to georeference the LiDAR point cloud according to Eq. (2). In this case, the platform orientation is transferred from the image BA results and the time stamps are transferred from the GPS data. Since both solutions are of much lower rate than the LiDAR data acquisition, interpolation is necessary. Finally, the accuracy of the point cloud will be then also dependent on the flight dynamics, and the chosen interpolation model.

\section{CONCLUSIONS AND FUTURE WORK}

This work showed initial results of the performance assessment of the UAS LiDAR point cloud obtained with the Velodyne sensor. The analysis was executed to assess the performance of dual-frequency GPS and medium-grade MEMS IMU sensors in the trajectory reconstruction for georeferencing LiDAR point cloud. The results showed that miniaturized dual-frequency GPS receivers are suitable for direct georeferencing of the UAS platform while some of the IMU sensors experience strong vibrations caused by platform motors that degrades their performance. The reduced IMU performance resulted in decreased accuracy of the LiDAR point cloud that was $0.49 \mathrm{~m}$ for the vertical component, and was much lower than the accuracy of the point cloud created from images taken during similar flights. The relative comparison between these point clouds showed that the accuracy of the horizontal component of the LiDAR point cloud is in the same magnitude as for vertical component. Although the LiDAR point cloud is sparser than image point cloud, in many situations, it is more complete as it allows to map dynamic scenarios and objects that lack of the texture necessary for dense image matching.

Reducing the impact of vibration, e.g. by adding mechanical dampers to the IMU mount will be investigated in the future. A promising result of accurate platform direct georeferencing was obtained by trajectory reconstruction based on imagery data. The georeferencing of the LiDAR point cloud based on the solution obtained by indirect georeferencing of images will be investigated in the future by performing simultaneous flight with light-weight Velodyne VLP-16 laser scanner and GoPro camera.

\section{REFERENCES}

Amon, P., Riegl, U., Rieger, P., Pfennigbauer, M., 2015. UAVbased laser scanning to meet special challenges in lidar surveying. Proceedings of the Geomatics Indaba 2015 conference, August 11-13, 2015, Ekurhuleni, Gauteng, South Africa, online.

El-Sheimy, N., 2009. Emerging MEMS IMU and its impact on mapping applications. Photogrammetric Week, Stuttgart, Germany.

Girardeau-Montaut, D., 2016. CloudCompare - Open Source project. Online: http://www.danielgm.net/cc/ (accessed 30.03.2016).

Glennie, C., Lichti, D.D., 2010. Static calibration and analysis of the Velodyne HDL-64E S2 for high accuracy mobile scanning. Remote Sensing, 2(6), pp.1610-1624.

Hirose, M., Xiao, Y., Zuo, Z., Kamat, V. R., Zekkos, D., Lynch, J., 2015. Implementation of UAV localization methods for a mobile post-earthquake monitoring system. 2015 IEEE Workshop on Environmental, Energy and Structural Monitoring Systems (EESMS), July 9-10, 2015, pp. 66-71.

Jekeli, C., 2001. Inertial navigation systems with geodetic applications. Walter de Gruyter.

Kuhnert, K.D., Kuhnert, L., 2013. Light-weight sensor package for precision $3 \mathrm{D}$ measurement with micro UAVs e. g. powerline monitoring. International Archives of the Photogrammetry, Remote Sensing and Spatial Information Sciences, XL-1/W2, pp. 235-40. 
Lin, Y., Hyyppä, J., Jaakkola, A., 2011. Mini-UAV-borne LIDAR for fine-scale mapping. IEEE Geoscience and Remote Sensing Letters, 8(3), pp. 426-430.

Mandlburger, G., Pfennigbauer, M., Riegl, U., Haring, A., Wieser, M., Glira, P., Winiwarter, L., 2015. Complementing airborne laser bathymetry with UAV-based lidar for capturing alluvial landscapes. Proceedings of SPIE 9637, Remote Sensing for Agriculture, Ecosystems, and Hydrology XVII, 96370A, October 14, 2015, Toulouse, France, pp. 1-14.

Nagai, M., Chen, T., Shibasaki, R., Kumagai, H., Ahmed, A., 2009. UAV-borne 3-D mapping system by multisensor integration. IEEE Transactions on Geoscience and Remote Sensing, 47(3), pp. 701-708.

Roca, D., Armesto, J., Lagüela, S., Díaz-Vilariño, L., 2014. LIDAR-equipped UAV for building information modelling. International Archives of the Photogrammetry, Remote Sensing and Spatial Information Sciences, XL-5, pp. 523-527.

Skaloud, J., Lichti, D., 2006. Rigorous approach to bore-sight self-calibration in airborne laser scanning. ISPRS Journal of Photogrammetry and Remote Sensing, 61(1), 47-59.

Toth, C., Jozkow, G., and Grejner-Brzezinska, D., 2015. Mapping with Small UAS: A Point Cloud Accuracy Assessment. Journal of Applied Geodesy, 9(4), pp. 213-226.

Tulldahl, H. M., Larsson, H., 2014. Lidar on small UAV for 3D mapping. Proceedings of SPIE Security+ Defence conference, International Society for Optics and Photonics, pp. 925009925009 .

Wallace, L., Lucieer, A., Watson, C., Turner, D., 2012. Development of a UAV-LiDAR system with application to forest inventory. Remote Sensing, 4(6), pp. 1519-1543.

Yang, B., Chen, C., 2015. Automatic registration of UAV-borne sequent images and LiDAR data. ISPRS Journal of Photogrammetry and Remote Sensing, 101, pp. 262-274.

Zhe, L., Yu, Y., Yabing, J., ShuGuang, Z., 2015. The Design and Testing of a LiDAR Platform for a UAV for Heritage Mapping. International Archives of the Photogrammetry, Remote Sensing and Spatial Information Sciences, XL-1/W4, pp, 17-24. 